\title{
Eficacia de la ivermectina en el tratamiento de niños colombianos parasitados por Strongyloides stercoralis
}

\author{
Leonardo Elías Ordóñez, Esther Sofía Angulo \\ Hospital Sagrado Corazón de Jesús, La Hormiga, Putumayo, Colombia.
}

Se llevó a cabo un estudio para determinar la eficacia de $200 \mu \mathrm{g} / \mathrm{kg}$ diarios de ivermectina por 2 días en niños con estrongiloidiasis no complicada de un poblado de la Amazonia colombiana. Los criterios de inclusión fueron: ausencia de enfermedad aguda actual, sin ingestión de antiparasitarios en el último mes y sin presencia de trastornos hepáticos ni neurológicos severos; además, los participantes debían tener, al menos, 2 muestras positivas de 4 para Strongyloides stercoralis procesadas con la técnica de Baermann; este último criterio con el fin de disminuir la frecuencia de falsos negativos en los exámenes de seguimiento. De los 60 participantes que cumplieron estos requisitos, sólo 49 completaron, al menos, 4 muestras de seguimiento y fueron incluidos en los resultados. El porcentaje de curación contra $S$. stercoralis fue del $93,8 \%$ (46/49), con efectos secundarios leves y pasajeros. Se describen, además, los resultados contra otros parásitos intestinales. En conclusión, de acuerdo con la información disponible, la dosis diaria de ivermectina de $200 \mu \mathrm{g} / \mathrm{kg}$ por 2 días es el esquema terapéutico recomendado para la estrongiloidiasis no complicada en niños.

Palabras clave: Strongyloides stercoralis, estrongiloidiasis, ivermectina, parásitos intestinales

Efficacy of ivermectin in the treatment of children parasitized by Strongyloides stercoralis

In a small village of Amazonian Coombia, the efficacy of ivermectin $(200 \mu \mathrm{g} / \mathrm{kg} / \mathrm{day})$ was determined in a two-day treatment of children with uncomplicated strongyloidiasis. Criteria for inclusion in the study were as follows: absence of acute disease, no pretreatment with antiparasitic drugs within the last month, absence of severe liver or neurological disorders, and at least 2 of 4 stool samples positive for Strongyloides stercoralis. The Baermann technique was used to detect larvae; it had the advantage of reducing the frequency of false negative results in the subsequent examinations. Of 60 potential subjects, 49 fulfilled the above criteria. The cure rate for the $S$. stercoralis infection was $94 \%$ (46/49), with slight and temporary side effects. The effects of ivermectin on other intestinal parasites were characterized as well. In conclusion, a $200 \mu \mathrm{g} / \mathrm{kg} / \mathrm{d}$ ivermectin dose was an adequate therapeutic regimen in the treatment of uncomplicated strongyloidiasis in children.

Key words: Strongyloides stercoralis, strongyloidiasis, ivermectin, intestinal parasites.

Para 1999, se estimaba que Strongyloides stercoralis parasitaba 70 millones de personas en el mundo, la mayoría de ellas en las zonas tropicales (1). Dos características sobresalientes de la estrongiloidiasis son la posibilidad de autoinfección y la presentación de cuadros de hiperinfección que producen una alta mortalidad,

\footnotetext{
Correspondencia:

Leonardo E. Ordóñez, Avenida 7 No. 151-67, apartamento 101, Bogotá; teléfono: (571) 6254356 leeoo2@hotmail.com
}

Recibido: 24/06/03; aceptado: 13/02/04 especialmente en pacientes inmunosuprimidos (2). En los últimos años se han definido algunos mecanismos por los cuales los parásitos intestinales, incluso S. stercoralis, pueden generar o agravar la desnutrición en niños, principalmente en los países en vía de desarrollo. El retraso del crecimiento en estatura es el parámetro más afectado $(3,4)$. Este último aspecto es, a nuestro juicio, el efecto nocivo del parasitismo intestinal más preocupante para la salud de las poblaciones.

Para el diagnóstico de la estrongiloidiasis se recomiendan dos técnicas: el método de 
Baermann y el cultivo en cajas de agar. La sensibilidad de los dos métodos es comparable en pacientes con cuadros no complicados (pacientes con cuadros crónicos sintomáticos o asintomáticos) cuando se examinan muestras seriadas y, en general, es superior al $70 \%$ con cuatro muestras $(5,6)$. Aunque se han desarrollado varias técnicas serológicas de elevada sensibilidad, presentan baja especificidad y un alto grado de reacciones cruzadas frente a otras parasitosis; por ello, actualmente se recomiendan para el tamizaje de poblaciones y de individuos en riesgo de desarrollar cuadros de hiperinfección, y en la evaluación postratamiento de la estrongiloidiasis (7). Algunos estudios recientes resaltan una característica importante de la estrongiloidiasis como es la variabilidad en la excreción de larvas en las heces de pacientes con infección sin complicaciones y las posibles implicaciones de este fenómeno en el diseño de estudios orientados a la determinación de la eficacia de determinado fármaco. Dreyer y colaboradores utilizaron el método de Baermann y encontraron que, en los pacientes con un resultado positivo de las cuatro muestras iniciales $(n=29)$, las cuatro muestras posteriores fueron negativas en el $75 \%$ de los casos sin ningún tipo de tratamiento (la sensibilidad de los exámenes de seguimiento fue del $25 \%$ ); sin embargo, en aquellos pacientes con 2, 3 o 4 muestras iniciales positivas ( $n=43)$, los resultados siguieron siendo positivos en $97,6 \%$ de los sujetos en, al menos, una de las cuatro muestras de seguimiento (8). Uparanukaw y colaboradores contaron las larvas expulsadas en los 8 días siguientes por los pacientes diagnosticados con estrongiloidiasis mediante cultivo en cajas con agar y encontraron que, aunque el resultado fue negativo uno o varios días (0 larvas/g de heces), en una muestra posterior se hallaron incluso varios miles de larvas/g de heces (un caso llegó a presentar 23.000 larvas/g de heces cuando dos días antes el conteo había sido de 0 larvas/g de heces) (9). La conclusión más importante de estas observaciones es que para la inclusión de individuos en un estudio para probar la eficacia de un fármaco contra $S$. stercoralis, se debe hacer un seguimiento que ofrezca el mínimo porcentaje posible de falsos negativos, lo cual se podría conseguir al seleccionar aquellos casos con más de dos muestras positivas de cuatro en los exámenes diagnósticos con la técnica de Baermann; de lo contrario, podríamos encontrar un porcentaje de curación con placebo de hasta el $75 \%$ (8).

La ivermectina es un medicamento semisintético derivado de las avermectinas, sustancias producidas por la fermentación del microorganismo Streptomyces avermitilis. La ivermectina tiene acción contra vermes y ectoparásitos que infectan animales y humanos. Este fármaco induce parálisis tónica en los nemátodos e insectos parásitos sensibles. Los estudios iniciales mostraron que, al parecer, actuaba como un potente agonista de los receptores del ácido gamma aminobutírico (GABA), un importante neurotransmisor; sin embargo, las investigaciones recientes señalan que su efecto puede estar mediado por potenciación, activación directa o por ambos mecanismos de los canales de cloro regulados por el glutatión y sensibles a la avermectina (10). Varios estudios han mostrado la eficacia de la ivermectina en el tratamiento de $S$.stercoralis, lo que sumado a la baja gravedad $y$ frecuencia de los efectos secundarios, lo convierten en una buena alternativa contra la estrongiloidiasis. De hecho, la Food and Drug Administration aprobó en los Estados Unidos su uso contra esta parasitosis en 1997.

El estudio inicial de Náquira en Perú mostró un porcentaje de curación del $94 \%$ con $150 \mu \mathrm{g} / \mathrm{kg}$ o $200 \mu \mathrm{g} / \mathrm{kg}$ de peso en dosis única, con un porcentaje de curación del $100 \%$ cuando se administraron $200 \mu \mathrm{g} / \mathrm{kg}$ en 2 días seguidos. Los estudios posteriores que compararon el efecto de la ivermectina con los dos fármacos previamente recomendados para el tratamiento de la estrongiloidiasis, el tiabendazol (12) y el albendazol $(13,14)$, mostraron la mayor eficacia del primero con una dosis doble de $200 \mu \mathrm{g} / \mathrm{kg}$ o única de 150$200 \mu \mathrm{g} / \mathrm{kg}$ de peso; los porcentajes de curación fueron de $100 \%, 83 \%$ y $82,9 \%$; mientras que el tiabendazol, $50 \mathrm{mg} / \mathrm{kg}$ diarios durante 3 días, mostró un porcentaje de curación de $89,4 \%$ y el albendazol, $400 \mathrm{mg} /$ día por 3 días, presentó porcentajes de curación de $38 \%$ y $45 \%$. En estos estudios, los efectos adversos en los pacientes tratados con ivermectina y albendazol fueron leves 
y pasajeros, mientras que con tiabendazol fueron acentuados y frecuentes (en el $95 \%$ de los que recibieron el medicamento) lo cual limita su uso. De acuerdo con estos resultados, la dosis diaria de $200 \mu \mathrm{g} / \mathrm{kg}$ por 2 días parece ser el esquema más adecuado para los casos no complicados. Incluso, en cuadros de hiperinfección de pacientes con sida, la ivermectina ha demostrado su eficacia con dosis repetidas de $200 \mu \mathrm{g} / \mathrm{kg}$ (15). Sin embargo, en algunos de estos estudios el seguimiento no fue adecuado y ninguno de ellos consideró en su diseño el impacto que puede tener en los resultados la variabilidad individual en la excreción de larvas; por lo tanto, consideramos necesario adelantar estudios adicionales que tengan en cuenta este aspecto para constatar los porcentajes de curación que ofrece el medicamento en la estrongiloidiasis no complicada; además, creemos que la población de estudio debe ser preferiblemente infantil, en quienes la erradicación del parásito puede conllevar importantes beneficios, especialmente de su estado nutricional.

Los objetivos del presente estudio fueron: 1) determinar la eficacia de la ivermectina en dosis diarias de $200 \mu \mathrm{g} / \mathrm{kg}$ durante dos días seguidos en niños con infección no complicada por $S$. stercoralis; 2) reportar el efecto del medicamento contra otros parásitos intestinales presentes en los participantes, y 3 ) determinar la frecuencia y la severidad de los efectos adversos asociados con el uso de la droga.

\section{Materiales y métodos}

\section{Área y sujetos de estudio}

El presente estudio se realizó entre agosto de 2001 y enero de 2002 en niños de dos escuelas públicas, la escuela La Parke y la escuela La Libertad, y niños de 2 a 7 años de los barrios aledaños a éstas, ubicadas en la localidad de La Hormiga, municipio Valle del Guamuez, departamento de Putumayo, Colombia; la descripción de sus características geográficas, socioeconómicas y demográficas se hizo en un estudio previo (16). Estos niños son objeto de las acciones que la oficina local de la Unicef adelanta en el municipio. El estudio se inició con 354 niños con edades entre los 2 y los 16 años, los cuales se sometieron a examen parasitológico por examen directo y método de Baermann modificado $(6,17)$. Se obtuvieron 4 muestras de cada participante; además, se obtuvieron los datos demográficos y nutricionales para calcular los índices antropométricos HAZ (height for age), WHZ (weight for height) y WAZ (weight for age), de acuerdo con las recomendaciones de la OMS $(18,19)$. Se obtuvo la información anterior de 237 sujetos; 112 de los 237 niños (47,3\%) tuvieron, al menos, una muestra positiva para $S$. stercoralis por el método de Baermann y 66 de ellos, 20 más muestras positivas. A estos 66 niños se les practicó un interrogatorio y un examen médico general con el fin de detectar alguno de los siguientes criterios de exclusión: 1) ingestión de cualquier antiparasitario en el último mes; 2) signos de enfermedad aguda actual; 3) trastornos neurológicos severos, y 4) trastornos hepáticos severos. Dos niños fueron excluidos por estar recibiendo antiparasitarios y un niño de 4 años que presentaba parálisis cerebral. Los padres de los 63 niños preseleccionados fueron informados verbalmente de los objetivos y los procedimientos que se iban a realizar durante el estudio y fueron invitados a participar; 60 de los 63 expresaron su deseo de ingresar al estudio y firmaron un consentimiento escrito; los 3 restantes se excluyeron porque planeaban salir de la región durante el periodo de seguimiento.

\section{Procedimientos de laboratorio}

Para el diagnóstico parasitológico, cada uno de los participantes recolectó 4 muestras de materia fecal en recipientes plásticos limpios, suministrados por el equipo investigador, con una capacidad aproximada de $180 \mathrm{ml}$, marcados con su nombre y un número de identificación; se les dieron instrucciones para recoger las muestras de heces en una cantidad mínima aproximada de $30 \mathrm{~g}$, las cuales se recolectaron con intervalos de 8 días, según fechas preestablecidas. Las muestras se observaron con microscopio de luz en examen directo; de cada una se prepararon dos láminas con solución salina normal y dos teñidas con lugol parasitológico. Además, todas las muestras se procesaron con el método de Baerman modificado, según la descripción hecha por Watson y colaboradores (17) y Kaminsky (6). 
Brevemente, se introducía totalmente en un frasco de sedimentación lleno de agua a $38^{\circ} \mathrm{C}$ una bolsa de gasa de 6 capas de grosor que contenía entre $30 \mathrm{~g}$ y $50 \mathrm{~g}$ de materia fecal. El agua no se recalienta para mantener la temperatura. La muestra se deja 2 horas, periodo en el cual las larvas, gracias a su termotropismo e hidrotropismo positivos, migran desde la materia fecal hacia el agua y se depositan en el fondo del frasco de sedimentación. Con una pipeta Pasteur, se extraen $10 \mathrm{ml}$ del fondo del frasco, se pasan a un tubo de centrífuga y se centrifugan a 2.000 rpm durante 5 min; se descarta el sobrenadante y con el sedimento se preparan láminas que se tiñen con lugol parasitológico y se procede a su lectura en el microscopio de luz. De cada muestra se observaron 4 láminas, como mínimo.

Adicionalmente, a cada sujeto se le practicó un recuento total de leucocitos (cámara de Neubauer), recuento porcentual de eosinófilos (coloración de Wright de extendido de sangre periférica) y concentración de hemoglobina (Microlab 200, software 1.0. Merk, Frankfurter, Alemania) en sangre venosa periférica.

En el seguimiento parasitológico, además del examen directo y la técnica de Baermann modificada, se usó el cultivo en papel filtro (Harada-Mori) que se leyó a las 72 y a las 120 horas. Se debe recordar que en esta última técnica se pueden encontrar además larvas filariformes de $S$. stercoralis y larvas rhabditiformes y filariformes de uncinarias (20). Se considera un resultado positivo la presencia de larvas rhabditiformes y filariformes de $S$. stercoralis con cualquiera de las técnicas parasitológicas descritas. Las características morfológicas utilizadas para la diferenciación de los estadios de las larvas y de las especies han sido descritas por otros autores; las diferencias en el primordio genital y en la cápsula bucal son las más relevantes para las larvas rhabditiformes $(20,21)$.

\section{Tratamiento}

Los 60 participantes recibieron ivermectina (Quanox $\AA$ en gotas, $200 \mu \mathrm{g} /$ gota) en dosis de $200 \mu \mathrm{g} / \mathrm{kg}$ de peso durante dos días consecutivos. El medicamento se administró en ayunas con un vaso de agua y en presencia de un miembro del equipo investigador. La presentación en gotas permitió administrar de forma más exacta la dosis a cada paciente. Tanto los niños como sus padres fueron instruidos para que reportaran posibles efectos adversos causados por el tratamiento y buscaran atención médica en el hospital local en caso necesario. También se les solicitó registrar por escrito la ingestión de cualquier fármaco durante el periodo de seguimiento, con el fin de establecer si había ingestión de algún otro antiparasitario.

Los restantes niños no incluidos en el estudio que presentaron parásitos intestinales, incluso los que sólo presentaron una muestra positiva para $S$. stercoralis, y los participantes del estudio al terminar el periodo de seguimiento fueron tratados según el siguiente esquema:

- Giardia lamblia y Entamoeba histolytical dispar: tinidazol, $60 \mathrm{mg} / \mathrm{kg}$ diarios por tres días

- Tricocéfalos: albendazol, 400 mg diarios por dos días

- Áscaris, uncinarias y oxiuros: albendazol, 400 mg diarios por un día; se repite a las tres semanas si hay ascariasis o uncinariasis.

- S. stercoralis: ivermectina, $200 \mu \mathrm{g} / \mathrm{kg}$ diarios por dos días.

\section{Seguimiento}

El seguimiento parasitológico se realizó las semanas 1, 2, 4, 6 y 8 luego de suministrado el tratamiento; cada muestra de materia fecal se analizó por examen directo, técnica de Baermann modificada y cultivo en papel de filtro. En estas mismas fechas, a cada niño se le practicó un recuento porcentual de eosinófilos en sangre venosa periférica. La evaluación de los efectos secundarios se hizo por medio de interrogatorio a los niños y sus padres los días 1 y 2 después del tratamiento y, posteriormente, cuando los niños debían llevar los especímenes de materia fecal; se permitió que expresaran libremente los síntomas asociados con la ingestión del fármaco y, además, se indagó la presencia de síntomas seleccionados de la literatura disponible. Los días 1 y 2 postratamiento, los niños y sus padres recibieron dos charlas educativas sobre la 
prevención del parasitismo intestinal, con énfasis en la utilización permanente de calzado para evitar la reinfección por $S$. stercoralis así como recomendaciones nutricionales generales.

Para la estrongiloidiasis, se definió su curación como la ausencia de larvas en 4 muestras de seguimiento, por lo menos, procesadas según las técnicas descritas.

\section{Resultados}

De los 60 niños que recibieron el tratamiento con ivermectina, 49 completaron el seguimiento programado (15 recogieron 4 muestras y 34 recogieron 5 muestras); los 11 restantes tuvieron seguimiento inadecuado. Los resultados sólo incluyen los 49 niños con adecuado seguimiento. Algunas de sus características demográficas y resultados de la valoración nutricional antes del tratamiento se muestran en el cuadro $1 \mathrm{y}$ en el cuadro 2, sus datos parasitológicos. La distribución por sexo muestra un leve predominio de hombres y la distribución por área de residencia muestra que la mayoría vivía en la cabecera urbana del municipio. Respecto a la valoración nutricional, se encontraron las siguientes frecuencias de índices antropométricos bajos: baja talla para la edad, $32,7 \%$; bajo peso para la talla, $11,1 \%$, y bajo peso para la edad, $20,4 \%$, frecuencias altas, según la clasificación propuesta por la OMS para valorar la severidad de la desnutrición en poblaciones (19). Este mal estado nutricional entre los participantes del estudio se debe tanto a procesos que conducen a bajo crecimiento en estatura como a aquéllos que conducen a no ganancia o pérdida de peso. Una niña de 12 años (hemoglobina, $11,9 \mathrm{~g} / \mathrm{dl}$ ) y un niño de 7 años (hemoglobina, 11,3 g/dl) presentaron anemia según los puntos de corte establecidos por la OMS para la concentración de hemoglobina: $11 \mathrm{~g} / \mathrm{dl}$ para niños entre 6 y 59 meses; $11,5 \mathrm{~g} / \mathrm{dl}$ para niños entre 5 y 11 años y $12 \mathrm{~g} / \mathrm{dl}$ para niños entre 12 y 14 años (22); los dos estaban parasitados, además de $S$. stercoralis, por uncinarias y giardias. El recuento de eosinófilos antes del tratamiento estuvo elevado $(>400 / \mu$ l en el recuento absoluto o mayor del $5 \%$ en el diferencial) en los 49 participantes (media: 2.280 eosinófilos/ $\mu \mathrm{l} ; 23,3 \%$ en el recuento diferencial), en dos pacientes llegó a representar más de la
Cuadro 1. Características demográficas y resultados de la valoración nutricional y de laboratorio en 49 niños colombianos con estrongiloidiasis.

\begin{tabular}{|c|c|}
\hline Variable & Valor \\
\hline $\begin{array}{l}\text { Rango de edad en meses (media) } \\
\text { Sexo }\end{array}$ & $31,6-197,7(113,7)$ \\
\hline $\begin{array}{l}\text { Sexo } \\
\text { Masculino } \\
\text { Femenino }\end{array}$ & $\begin{array}{l}28 / 49(57,1 \%) \\
21 / 49(42,8 \%)\end{array}$ \\
\hline $\begin{array}{l}\text { Área de residencia } \\
\text { Urbana } \\
\text { Rural }\end{array}$ & $\begin{array}{r}44 / 49(89,8 \%) \\
5 / 49(10,2 \%)\end{array}$ \\
\hline $\begin{array}{l}\text { Bajos índices antropométricos * } \\
\text { Baja talla para la edad }{ }^{1} \\
\text { Bajo peso para la talla }{ }^{2} \\
\text { Bajo peso para la edad }{ }^{3}\end{array}$ & $\begin{array}{r}16 / 49(32,7 \%) \\
2 / 31(11,1 \%) \\
10 / 49(20,4 \%)\end{array}$ \\
\hline $\begin{array}{l}\text { Concentración de hemoglobina }(\mathrm{g} / \\
\text { Rango } \\
\text { Media }\end{array}$ & $\begin{array}{r}11,3-15,3 \\
13,2\end{array}$ \\
\hline $\begin{array}{l}\text { Recuento de leucocitos (miles/ } \mu \mathrm{l} \text { ) } \\
\text { Rango } \\
\text { Media }\end{array}$ & $\begin{array}{r}7.000-14.300 \\
9.349\end{array}$ \\
\hline $\begin{array}{l}\text { Recuento de eosinófilos } \\
\text { Porcentaje, rango (media) } \\
\text { Valor absoluto/ } \mu \text { l, rango (media) }\end{array}$ & $\begin{array}{r}5 \%-55 \%(23,3 \%) \\
405-6.655(2.280,7)\end{array}$ \\
\hline
\end{tabular}

* Definidos como aquellos valores que se encuentren 2 desviaciones estándar o más por debajo de la media de referencia internacional recomendada por la NCHS/OMS, aplicable para todos los índices.

1 reducción del crecimiento en talla (stunting), valorado a través del índice HAZ, calculado para todos los participantes.

2 adelgazamiento (wasting), valorado a través del índice WHZ, calculado para 31 participantes que se encontraban dentro del rango de edad requerido (niños menores de 138 meses y niñas menores de 120 meses).

3 bajo peso (underweight), valorado a través del índice WAZ, calculado para todos los participantes.

mitad de los leucocitos circulantes (55\% y $51 \%$ ). La media del porcentaje de eosinófilos entre el grupo de participantes mostró una progresiva disminución luego de suministrado el tratamiento, el cual llegó a 8,36\% al término del periodo de seguimiento (figura 1). Aparte de $S$. stercoralis los parásitos más frecuentes en estos niños fueron: giardias, 38/49 (77,6\%); uncinarias, $27 / 49(55,1 \%)$; tricocéfalos, $7 / 49(14,3 \%)$, y áscaris, $5 / 49(10,2 \%)$; además, 45 de los 49 presentaron dos o más parásitos intestinales $(91,8 \%)$.

El porcentaje de curación con $200 \mu \mathrm{g} / \mathrm{kg}$ diarios de ivermectina por 2 días contra S. stercoralis fue 
Cuadro 2. Resultados parasitológicos de 49 niños colombianos con estrongiloidiasis, utilizando 4 muestras de materia fecal y analizadas por examen directo y el método de Baermann modificado.

\begin{tabular}{lr}
\hline Variable & Frecuencia \\
\hline Parásitos intestinales & \\
Giardias & $38 / 49(77,6 \%)$ \\
Uncinarias & $27 / 49(55,1 \%)$ \\
Tricocéfalos & $7 / 49(14,3 \%)$ \\
Ascaris & $5 / 49(10,2 \%)$ \\
E. histolytica/dispar & $2 / 49(4,1 \%)$ \\
Oxiuros & $1 / 49(2 \%)$ \\
Número de parásitos & \\
1 & $4 / 49(8,2 \%)$ \\
2 & $16 / 49(32,7 \%)$ \\
3 & $24 / 49(49 \%)$ \\
4 & $4 / 49(8,2 \%)$ \\
5 & $1 / 49(2 \%)$ \\
Poliparasitismo intestinal * & $45 / 49(91,8 \%)$ \\
Número de resultados positivos para & \\
$S$. stercoralis utilizando el método de Baermann \\
y 4 muestras & $19 / 49(38,8 \%)$ \\
2 & $12 / 49(24,5 \%)$ \\
3 & $18 / 49(36,7 \%)$ \\
4 & \\
\hline * Presencia de dos o más de los & parásitos antes \\
mencionados; incluye a $S$ stercoralis. & \\
&
\end{tabular}

de $93,8 \%$ (46/49). Los tres participantes en quienes el parásito persistió tuvieron cada uno un resultado positivo para $S$. stercoralis con la técnica de Baermann, dos de ellos una semana luego de suministrado el tratamiento y el tercero a las 8 semanas postratamiento; el frotis directo y el cultivo en papel filtro no detectaron el parásito en ninguno de los tres casos. Los porcentajes de curación contra $S$. stercoralis y otros parásitos intestinales en los niños del estudio se presentan en el cuadro 3. Todos los sujetos que presentaron áscaris, tricocéfalos, oxiuros y E. histolytica/ dispar tuvieron negativas las 4 muestras de seguimiento para estos parásitos; adicionalmente, en 21 de los $38(55,3 \%)$ que presentaban giardias y en 8 de los $27(29,6 \%)$ que presentaban uncinarias, estos parásitos no estuvieron presentes en las muestras de seguimiento.

Respecto a los efectos secundarios asociados con la ingestión de ivermectina experimentados por los participantes, encontramos que la distensión

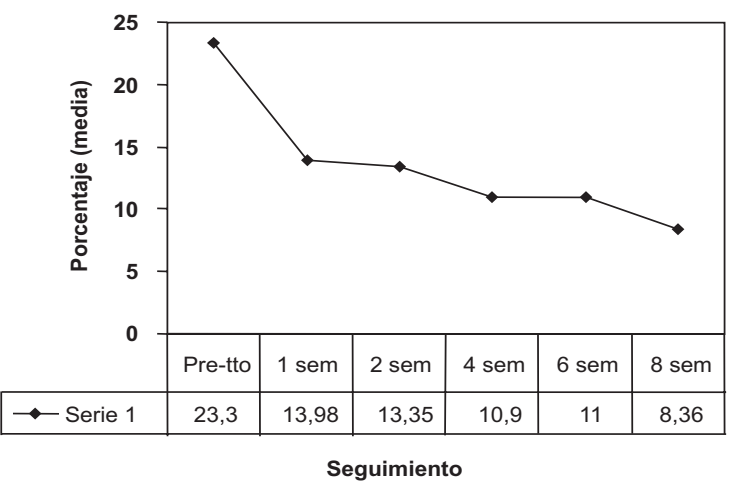

*Recuento realizado en frotis de sangre venosa periférica

Figura 1. Media del recuento porcentual de eosinófilos en frotis de sangre periférica de 49 niños, antes y después de recibir tratamiento con ivermectina.

Cuadro 3. Porcentajes de curación contra $S$. stercoralis y otros parásitos intestinales en 49 niños colombianos que recibieron $200 \mu \mathrm{g} / \mathrm{kg}$ diarios de ivermectina por dos días.

Parásito

Porcentaje de curación

Strongyloides stercoralis

$93,8 \%(46 / 49)$

Uncinarias

$29,6 \%(8 / 27)$

Giardias

$55,3 \%(21 / 38)$

abdominal fue el más frecuente (6/49), seguido por el vértigo (4/49), las náuseas (4/49) y las deposiciones blandas o líquidas (4/49); 10 de los $49(20,4 \%)$ reportaron la presencia de uno o más efectos secundarios. Todos se presentaron entre 2 y 72 horas después de la administración de la primera dosis del medicamento, la intensidad fue leve en todos los casos (10/10) y no produjo limitación en la realización de sus actividades diarias. Los síntomas reportados como frecuentes en otros estudios, como cefalea, tos y fiebre, no se presentaron en ninguno de los participantes del estudio.

Los 60 participantes que ingresaron al estudio recibieron además de los antiparasitarios un suplemento multivitamínico oral (Ferry-C Fol ${ }^{\circledR}$, jarabe); a los dos niños que presentaron anemia también se les suministró diariamente sulfato ferroso, $2 \mathrm{mg} / \mathrm{kg}$ repartido en dos dosis, más ácido fólico $1 \mathrm{mg}$; a cada uno se les entregaron suficientes dosis para 3 meses de tratamiento. 


\section{Discusión}

S. stercoralis es un parásito relativamente olvidado tanto en el campo investigativo como en los programas de control; el escaso e incompleto conocimiento de su frecuencia en las diferentes regiones del mundo y de las consecuencias individuales y de población que produce su presencia lo han conducido a este punto. Sin duda alguna, las dificultades que presenta su diagnóstico parasitológico por lo dispendioso de las técnicas recomendadas y la necesidad de procesar varias muestras son las responsables en gran parte de la imprecisión en la importancia que para la salud pública representa la estrongiloidiasis. Un ejemplo que ilustra este punto lo observamos en la región del Valle del Guamuez, Putumayo; un estudio realizado en el 2000 con población hospitalaria, mediante el examen directo de las heces con una sola muestra como método diagnóstico, mostró que entre los niños de 4 a 15 años la frecuencia de S. stercoralis era del $7,7 \%$, el cuarto helminto en frecuencia luego de uncinarias, tricocéfalos y áscaris. Sin embargo, la frecuencia de este parásito reportada en un estudio posterior fue del $47,3 \%$ en niños de un rango de edad comparable y se constituye en el helminto más frecuente (23). En este último estudio, la sensibilidad de los métodos para el diagnóstico de $S$. stercoralis, áscaris, tricocéfalos, y uncinarias es comparable y, como se describió en otros estudios, las características culturales, socioeconómicas y geográficas de la región son bastante homogéneas $(16,23)$; con estas consideraciones en mente, podemos suponer que la estrongiloidiasis es un problema relevante para la salud pública de esta región, hecho que no se había puesto de manifiesto antes por la subvaloración que resulta del uso de métodos con poca sensibilidad.

Es conveniente mencionar que el cultivo en cajas de agar es una herramienta diagnóstica menos dispendiosa y con sensibilidad similar al método de Baermann $(5,6,21)$; el empleo rutinario de este método en instituciones de moderada complejidad ayudaría a comprender mejor la epidemiología de esta parasitosis.

Los resultados del presente estudio confirman la eficacia de la ivermectina en la población infantil con cuadros de estrongiloidiasis no complicada. A diferencia de estudios previos, el porcentaje de curación se aleja un poco del $100 \%$ cuando se utiliza la dosis diaria de $200 \mu \mathrm{g} / \mathrm{kg}$ por dos días consecutivos, 93,7\% en este estudio; los criterios de selección utilizados, encaminados a disminuir la frecuencia de falsos negativos (sujetos con, al menos, 2 muestras positivas para $S$. stercoralis de 4 procesadas por la técnica de Baermann) probablemente explican este hecho. Hay un punto por discutir respecto a los tres niños en quienes persistió $S$. stercoralis luego de recibir la ivermectina; dos de ellos tuvieron una muestra positiva la semana 1 de seguimiento y constituyen un fracaso del tratamiento; sin embargo, el tercero presentó una muestra positiva la semana 8 de seguimiento y, además del fracaso del tratamiento, se debe considerar la alternativa de la reinfección. Esto es posible porque los niños continuaron habitando la misma región de alta prevalencia para esta parasitosis; además, si bien se dieron recomendaciones tendientes a evitar la reinfección, no es posible constatar su cumplimiento; finalmente, los estudios experimentales en perros, un modelo con comportamiento similar a los humanos, muestran que en la historia natural de la parasitosis se encuentran larvas en las heces luego de 2 a 3 semanas de la infección (22-24).

Sin embargo, falta determinar si los porcentajes de curación de las dosis únicas de ivermectina, especialmente la de $200 \mu \mathrm{g} / \mathrm{kg}$ por día, también disminuyen al incluir en el diseño criterios de selección similares y, si es así, cuál es la magnitud de esta diferencia y determinar la importancia de este efecto a la hora de hacer una recomendación posológica.

Los efectos adversos asociados con la ingestión del medicamento fueron leves y pasajeros, similar a los estudios previos; incluso, la frecuencia de estos efectos no fue muy diferente de la de aquellos estudios en que se utilizó la dosis única.

Con respecto a la erradicación de otros parásitos intestinales, encontramos que para las uncinarias el porcentaje de curación fue del $29,6 \%$, no muy diferente del encontrado por Náquira et al. de $22 \%$ con la misma dosis (11), aunque sí difiere del estudio realizado por Marti et al. de 0\% cuando se utilizó la dosis única (14). Debido al escaso 
número de participantes con tricocéfalos, 7 , áscaris, 5, oxiuros, 1, y E. histolytica/dispar, 2, los porcentajes de curación del $100 \%$ en los tres casos son difíciles de interpretar, aunque la alta eficacia del medicamento contra estos helmintos es reconocida por Náquira y Marti $(11,14)$. Llama la atención el porcentaje de curación del 55,3\% de giardiasis en los niños después de recibir el medicamento, efecto no descrito en la literatura que consultamos. Hablar de un efecto del medicamento contra el protozoario es precipitado debido a la falta de un grupo de control en el estudio y al desconocimiento del porcentaje de curación espontánea en la población estudiada; dado que se descartó con relativa seguridad la ingestión de otro antiparasitario, se abre la puerta para evaluar este aspecto en estudios posteriores.

En un estudio previo realizado por Gann (12) se encontró que la eosinofilia asociada con la estrongiloidiasis disminuyó en el grupo total, un mes después de recibir tratamiento con ivermectina o tiabendazol; en nuestro estudio, al cabo de las 8 semanas de seguimiento, sólo en 9 de los 49 sujetos el recuento porcentual de eosinófilos llegó a valores normales $(<5 \%)$, hecho probablemente causado por la persistencia de otros parásitos intestinales en la población de estudio. Sin embargo, la progresiva disminución en el recuento porcentual de eosinófilos puede ser un elemento útil en el seguimiento clínico postratamiento.

En conclusión, encontramos que la ivermectina en dosis diarias de $200 \mu \mathrm{g} / \mathrm{kg}$ por dos días consecutivos tiene una alta eficacia y buena tolerancia en niños con estrongiloidiasis no complicada y, teniendo en cuenta la información disponible, consideramos que es el esquema terapéutico de elección en estos casos. Adicionalmente, llamamos la atención para incorporar el tratamiento de esta parasitosis en los programas de control en salud pública, especialmente en población infantil, en aquellas regiones en donde se conozca o existan las condiciones para que $S$. stercoralis tenga una alta prevalencia.

\section{Agradecimientos}

Agradecemos a los participantes, padres de familia, rectores de las escuelas y líderes comunitarios por su colaboración en el desarrollo de este trabajo. También, la colaboración del director del Hospital Sagrado Corazón de Jesús, en La Hormiga, Putumayo, doctor José Víctor Pérez, por facilitarnos las instalaciones necesarias para el procesamiento de las muestras y la ayuda en la consecución de los antiparasitarios. A la oficina local de la Unicef La Hormiga, por colaborar en la coordinación y ejecución del proyecto. A las enfermeras jefes Marcelina Cuarán Inagán y Arabelly Montoya, quienes participaron activamente en el trabajo de campo. Al laboratorio farmacéutico Dermacare, S.A. por suministrar su producto Quanox ® gotas, utilizado como parte del tratamiento antiparasitario; al laboratorio Bremyg que nos suministró su producto Ferry-CFol $\circledast$ jarabe, multivitamínico entregado a los participantes del estudio.

\section{Referencias}

1. Crompton DWT. How much human helminthiasis is there in the world? J Parasitol 1999;85:397-403.

2. Mahmoud AAF. Strongyloidiasis. Clin Infect Dis 1996; 23:949-53.

3. Stephenson LS, Latham MC, Ottesen EA. Malnutrition and parasitic helminth infections. Parasitology 2000; 121(suppl):s23-s38.

4. Solomons NW. Pathways to the impairment of human nutritional status by gastrointestinal pathogens. Parasitology 1993;107(suppl):s19-s35.

5. Sato Y, Kobayashi J, Toma H, Shiroma Y. Efficacy of stool examination for detection for Strongyloides infection. Am J Trop Med Hyg 1995;53:248-50.

6. Kaminsky RG. Evaluation of three methods for laboratory diagnosis of Strongyloides stercoralis infection. J Parasitol 1993;79:277-80.

7. Sato Y, Kobayashi J, Shiroma Y. Serodiagnosis of strongyloidiasis. The application and significance. Rev Inst Med Trop Sao Paulo 1995;37:35-41.

8. Dreyer G, Fernandez-Silva E, Alvez S, Rocha A, Albuquerque R, Addiss 0 . Patterns of detection of Strongyloides stercoralis in stool specimens: implications for diagnosis and clinical trials. J Clin Microbiol 1996;34:2569-71.

9. Uparanukaw P, Phonngsri S, Morakote N. Fluctuations of larvae excretion in Strongyloides stercoralis infection. Am J Trop Med Hyg 1999;60:1967-73.

10. Tracy JW, Webster LT. Fármacos utilizados en la quimioterapia de las helminthiasis. En: Goodman y Gilman, editores. Las bases farmacológicas de la 
terapéutica. Novena edición. México: McGraw-Hill interamericana; 1996. p.1073-91.

11. Náquira C, Jiménez G, Guerra JG, Bernal R, Nalyn $\mathrm{D}$, Neu D, et al. Ivermectin for human strongyloidiasis and other intestinal helminths. Am J Trop Med Hyg 1989; 40:304-9.

12. Gann PH, Neva FA, Gam AA. A randomized trial of single and two-dose ivermectin versus thiabendazole for treatment of strongyloidiasis. J Infect Dis 1994;169: 1076-9.

13. Datry A, Hilmarsdottir I, Mayorga-Sagastume R, Lyagoubi H, Gaxotte T, Biligui S, et al. Treatment of Strongyloides stercoralis infection with ivermectin compared with albendazole: results of an open study of 60 cases. Trans R Soc Trop Med Hyg 1994;88:344-5.

14. Marti H, Haji H, Savili L, Shwaya HM, Mgent FA, Ameir SJ, et al. A comparative trial of a single-dose ivermectin versus three days of albendazole for treatment of Strongyloides stercoralis and other soil-transmitted helmiths infections in children. Am J Trop Med Hyg 1999;55:477-81.

15. Torres JR, Isturiz R, Murillo J, Guzman M, Contreras R. Efficacy of ivermectin in the treatment of strongyloidiasis complicating AIDS. Clin Infect Dis 1993;17:900-2.

16. Ordóñez LE, Angulo ES. Parasitismo intestinal en el Valle del Guamuez y San Miguel, Putumayo, Colombia. Medicina y Laboratorio 2000;9:565-74.

17. Watson JM, Al-Hafidh R. A modification of the Baermann funnel technique, and its use in establishing the infection potential of human hookworms carriers. Ann Trop Med Parasitol 1957;41:15-6.
18. WHO Working Group. Use and interpretation of anthropometric indicators of nutritional status. Bull WHO 1986;64:929-41.

19. Gorstein J, Sullivan K, Yip R, De Onis M, Trowbridge $P$, Fajans $P$, et al. Issues in the assessment of nutrition status using anthropometry. Bull WHO 1994;72:273-83.

20. Organización Panamericana de la Salud. Parasitología. En: Manual de técnicas básicas para un laboratorio de salud. Publicación científica No 439. Washington, D.C.: Organización Panamericana de la Salud; 1983. p.168-9.

21. Botero D, Restrepo M. Técnicas de laboratorio en parasitología médica. En: Botero D, Restrepo M, editores. Parasitosis humanas. Cuarta edición. Medellín: CIB; 2003. p.467.

22. Stephenson LS, Latham MC, Ottesen EA. Global malnutrition. Parasitology 2000;121(suppl):s5-s22.

23. Ordóñez LE, Angulo ES. Desnutrición y parasitismo intestinal en niños de una población de la Amazonia colombiana. Biomédica 2002;22:486-98.

24. Grove DI, Northern C. Infection and immunity in dogs infected with a human strain of Strongyloides stercoralis. Trans R Soc Trop Med Hyg 1982;76:833-8.

25. Grove DI, Heenan PJ, Northern C. Persistent and disseminated infections with Strongyloides stercoralis in immunosuppressed dogs. Intern J Parasitol 1983;13: 483-90.

26. Genta RM, Schad GA, Hellman ME. Strongyloides stercoralis: parasitological, immunological and pathological observations in immunosuppressed dogs. Trans R Soc Trop Med Hyg 1986;80:34-41. 